\title{
Caracterização físico-mecânica de compósitos porosos de hidroxiapatita- titânia confeccionados pelo método da esponja polimérica
}

\section{(Physical-mechanical characterization of hydroxyapatite-titanium oxide composites prepared by the polymeric sponge method)}

\author{
A. G. S. Galdino ${ }^{1,2}$, C. A. C. Zavaglia ${ }^{2}$ \\ ${ }^{1}$ Fatec Mogi Mirim \\ ${ }^{2}$ Faculdade de Engenharia Mecânica, UNICAMP, R. Mendeleyev 200, Cidade Universitária Zeferino Vaz, \\ Campinas, SP 13083-860 \\ andreg28@gmail.com,zavagl@fem.unicamp.br
}

\begin{abstract}
Resumo
Ao longo das últimas décadas as biocerâmicas vem sendo utilizadas como materiais para reconstrução óssea, na qual a hidroxiapatita é uma das biocerâmicas mais utilizadas. Entretanto, a resistência mecânica da hidroxiapatita não é tão alta quando comparada com outras biocerâmicas. Este trabalho teve como objetivo fazer a caracterização físico-mecânica de compósitos de $\mathrm{HA}^{-\mathrm{TiO}}{ }_{2}$ porosos. As amostras de $\mathrm{HA}-\mathrm{TiO}_{2}$ foram confeccionadas pelo método da esponja polimérica nas proporções de $70 \%-30 \%, 60 \%$ - $40 \%$ e $50 \%$ - $50 \% \mathrm{HA}$ - $\mathrm{TiO}_{2}$, calcinadas a $550{ }^{\circ} \mathrm{C}$ para retirada da esponja polimérica e sinterizadas a $1250{ }^{\circ} \mathrm{C}, 1300{ }^{\circ} \mathrm{C}$ e $1350{ }^{\circ} \mathrm{C}$. As amostras foram submetidas a ensaios mecânicos de compressão e dureza Vickers e a ensaios físicos de absorção de água, densidade aparente, retração linear de queima e porosidade aparente. Os resultados mostraram ser relativamente melhores quando comparados com os da hidroxiapatita, e estão de acordo com os encontrados na literatura.
\end{abstract}

Palavras-chave: compósitos biocerâmicos, caracterização físico-mecânica, reconstrução óssea.

Abstract

Bioceramics have been used as bone reconstruction materials in the last decades, hydroxyapatite being one of the most used for this purpose. However, the hydroxyapatite mechanical strength is not so high when compared to other bioceramics. This work aimed on characterizing physically and mechanically $\mathrm{HA}-\mathrm{TiO} \mathrm{O}_{2}$ composites. Samples were prepared by the polymeric sponge method with $70 \%-30 \% \mathrm{wt}$., $60 \%-40 \% \mathrm{wt}$. and $50 \%-50 \% \mathrm{wt}$. of $\mathrm{HA}-\mathrm{TiO}$, calcined at $550^{\circ} \mathrm{C}$ for sponge burning out and sintered at $1250{ }^{\circ} \mathrm{C}$, $1300{ }^{\circ} \mathrm{C}$ and $1350{ }^{\circ} \mathrm{C}$. The samples were submitted to mechanical essays of compression and Vickers hardness and to physical essays of water absorption, apparent density, burning linear retraction and apparent density. Results showed relatively better than those of pure hydroxyapatite and they are in agreement with the literature.

Keywords: bioceramic composites, physical mechanical characterization, bone reconstruction.

\section{INTRODUÇÃO}

Os biomateriais cerâmicos são cada vez mais importantes na clínica médica para a regeneração do tecido ósseo e, mais recentemente, vem sendo utilizados como carreadores de células nas terapias celulares. Um grande desafio da pesquisa atual reside em produzir biomateriais comerciais com características físico-químicas e morfológicas que estimulem a osteogênese. Dessa forma, propicia-se a formação de estruturas semelhantes aos elementos de matriz extracelular, facilitando a mobilização, expansão e integração de populações de células regenerativas, fomentando o reparo de lesões ou a renovação de tecidos degenerados. Os biomateriais substitutos ósseos podem ser de origem natural ou sintética. A vantagem da utilização de materiais de origem natural - tais como o xenoenxerto de origem bovina deve-se à estrutura biológica única, não reprodutível nos materiais de origem sintética. Destaca-se ainda que os xenoenxertos inorgânicos (livres de proteínas ou outros resíduos orgânicos) apresentam-se como um excelente material osteocondutor quando adequadamente processados. A presença dos íons de carbonatos na estrutura da apatita dos xenoenxertos atribui a estes biomateriais uma biodegradabilidade não usualmente encontrada nos equivalentes sintéticos. Junto a isto, deve-se considerar outra qualidade dos xenoenxertos: seu processamento adequado possibilita reproduzir com fidelidade a morfologia natural do osso humano $[1,2]$.

Dentre os materiais utilizados, a hidroxiapatita (fosfato de cálcio - $\mathrm{Ca}_{10}\left(\mathrm{PO}_{4}\right)_{6}(\mathrm{OH})$ ) é o mais utilizado, por ser idêntico ao material encontrado nos ossos, sendo aplicável para engenharia do tecido ósseo. Porém, este material possui resposta fraca sob tensão mecânica [3, 4]. A hidroxiapatita foi usada inicialmente como pó, em pequenos implantes sem 
carga, revestimentos, implantes porosos para crescimento ósseo. Pode facilmente ser degradada se as condições forem favoráveis ( $\mathrm{pH}$, ataque químico, fagocitose) e no estado puro, degrada rapidamente. Devido à fraca resposta sob tensão mecânica da hidroxiapatita, vários compósitos têm sido desenvolvidos a fim de aumentar a resposta da hidroxiapatita quando submetida a tensões mecânicas [3, 4]. Há vários métodos que indicam formas de se confeccionar corpos porosos de materiais cerâmicos [5-10].

Este trabalho tem por objetivo avaliar as propriedades físico-mecânicas de compósitos porosos de hidroxiapatita - titânia (HA-TiO ${ }_{2}$ em três composições diferentes, confeccionados pelo método da esponja polimérica [8] na obtenção de scaffolds para engenharia tecidual óssea.

\section{MATERIAIS E MÉTODOS}

A hidroxiapatita (HA) utilizada neste trabalho foi gentilmente cedida pela empresa Inside Materiais Avançados Ltda., Belo Horizonte, MG. Foram realizados ensaios de fluorescência de raios $\mathrm{X}$ com o intuito de identificar o percentual de elementos químicos, além das temperaturas onde ocorreu mudança de fases. A análise de fluorescência de raios X foi feita em um espectrômetro Rigaku RIX 3100. Os percentuais dos elementos químicos na hidroxiapatita

Tabela I - Resultados de análise de fluorescência de raios $\mathrm{X}$ da hidroxiapatita, como recebida.

(Table I-X-rayfluorescence results of hydroxyapatite as received.)

\begin{tabular}{cc}
\hline Componente & Quantidade em massa (\%) \\
\hline $\mathrm{O}$ & 45,42 \\
$\mathrm{Ca}$ & 37,17 \\
$\mathrm{P}$ & 17,02 \\
$\mathrm{Mg}$ & 0,13 \\
$\mathrm{Sr}$ & 0,08 \\
$\mathrm{Si}$ & 0,01 \\
$\mathrm{Al}$ & 0,045 \\
$\mathrm{Fe}$ & 0,02 \\
$\mathrm{~S}$ & 0,02 \\
$\mathrm{Na}$ & 0,02 \\
$\mathrm{~K}$ & 0,01 \\
$\mathrm{Ni}$ & 0,005 \\
\hline
\end{tabular}

estão indicados na Tabela I.

Foram feitas análises de fluorescência de raios X, difração de raios $\mathrm{X}$ e análise termogravimétrica na amostra de titânia, com o intuito de identificar o percentual de elementos químicos, além das temperaturas onde ocorreu mudança de fases. Os resultados estão indicados na Tabela II.

A esponja polimérica utilizada foi de poliuretano comercial e a sua estrutura de poros pode ser vista na Fig. 1.

Foram utilizados bicarbonato de sódio $\mathrm{NaHCO}_{3}$ Merck, álcool comercial Cooperalcool INPM 92,8º água destilada.
Tabela II - Resultados de fluorescência de raios X para a titânia, como recebida.

(Table II - X-ray fluorescence results for titanium oxide as received.)

\begin{tabular}{cc}
\hline Componente & Quantidade em massa (\%) \\
\hline $\mathrm{TiO}_{2}$ & 95,08 \\
$\mathrm{Al}_{2} \mathrm{O}_{3}$ & 3,586 \\
$\mathrm{SiO}_{2}$ & 1,106 \\
$\mathrm{Na}_{2} \mathrm{O}$ & 0,115 \\
$\mathrm{SO}_{3}$ & 0,033 \\
$\mathrm{CaO}$ & 0,028 \\
$\mathrm{P}_{2} \mathrm{O}_{5}$ & 0,023 \\
$\mathrm{Fe}_{2} \mathrm{O}_{3}$ & 0,009 \\
$\mathrm{NiO}$ & 0,009 \\
$\mathrm{As}_{2} \mathrm{O}_{3}$ & 0,007 \\
\hline
\end{tabular}
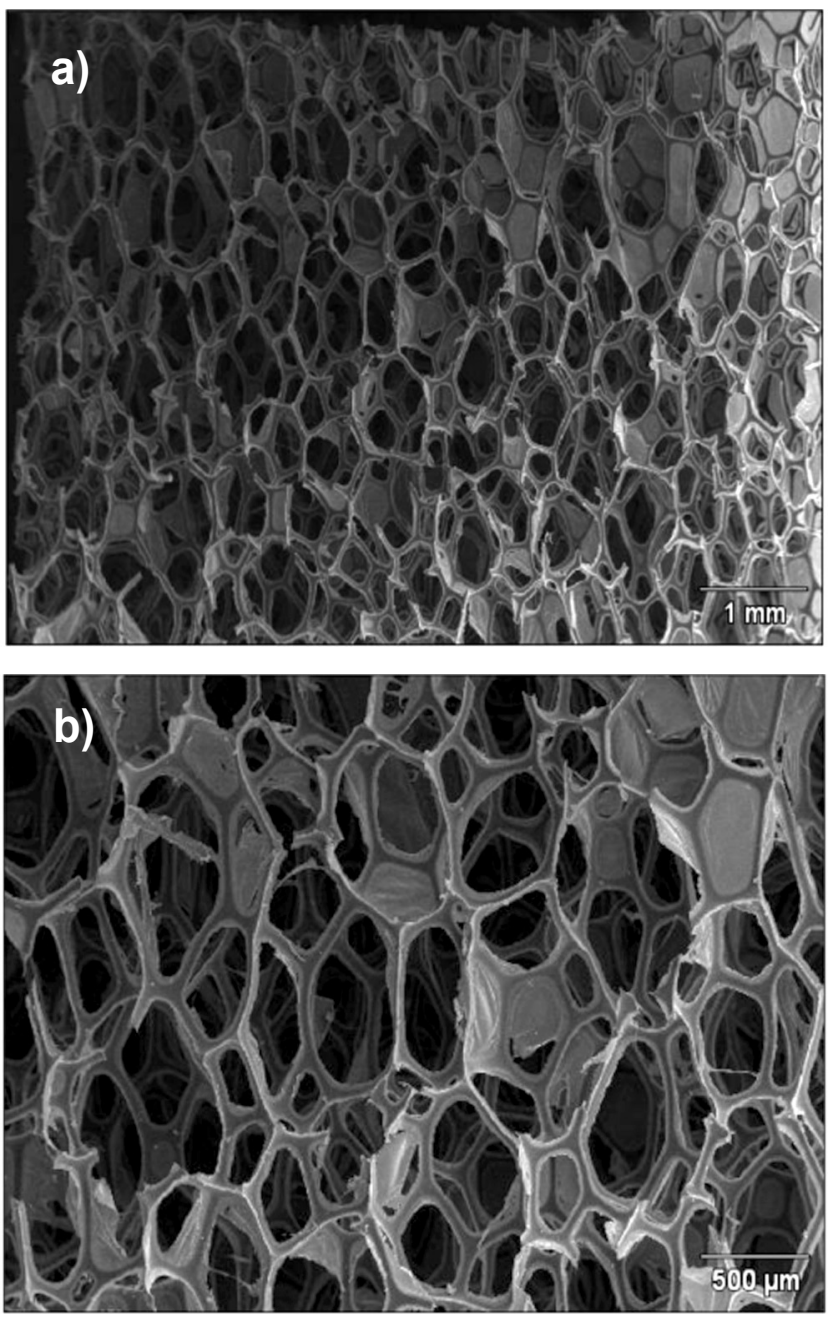

Figura 1: Distribuição e forma dos poros da esponja comercial de poliuretano; (a) visão geral da esponja (aumento de 15x), (b) geometria dos poros.

(Figure 1: Porous distribution and shape of commercial polyurethane sponge; (a) general view of the sponge (15x), (b) porous geometry.) 
Inicialmente, a hidroxiapatita e a titânia, juntamente com o bicarbonato de sódio, foram misturados e peneirados em peneira ABNT com $710 \mu \mathrm{m}$ de abertura. O bicarbonato de sódio foi misturado ao compósito por ser um agente ligante e floculante. Três porções do compósito foram preparadas, a saber: $50 \% \mathrm{HA}-50 \% \mathrm{TiO}_{2}(\operatorname{amostra} \mathrm{A}), 60 \% \mathrm{HA}-40 \% \mathrm{TiO}_{2}$ (B) e $70 \% \mathrm{HA}-30 \% \mathrm{TiO}_{2}(\mathrm{C})$. Também foram confeccionados corpos de prova de hidroxiapatita com bicarbonato de sódio, pelo método de esponja polimérica [8], com dimensões 5 $\mathrm{mm}$ de diâmetro por $12,5 \mathrm{~mm}$ de altura para os ensaios de compressão. Em todos os casos foram confeccionados 5 corpos de prova para avaliação. Estes foram aquecidos a 550 ${ }^{\circ} \mathrm{C}$ a $0,5^{\circ} \mathrm{C} / \mathrm{min}$ para a retirada da esponja polimérica e, em seguida, sinterizados a $1250{ }^{\circ} \mathrm{C}, 1300{ }^{\circ} \mathrm{C}$ e $1350{ }^{\circ} \mathrm{C}$ a $10^{\circ} \mathrm{C} /$ min, com patamar de $2 \mathrm{~h}$. A retirada da esponja polimérica e a sinterização dos corpos de prova foram feitas em fornos, com resfriamento até a temperatura ambiente. Para os ensaios de dureza Vickers foram confeccionados corpos de prova com dimensões $12 \mathrm{~mm}$ de diâmetro por $20 \mathrm{~mm}$ de altura com pressão de $10 \mathrm{MPa}$, para que estes estivessem porosos. Os corpos de prova foram submetidos a ensaios tecnológicos, de acordo com metodologia descrita por Souza Santos [11]. As características cerâmicas observadas foram: porosidade aparente (PA), absorção de água (AA), retração linear de queima (RQ) e densidade aparente (DA). Os resultados foram obtidos pela média aritmética de 5 corpos de prova e foram calculadas pelas equações A, B, C e $\mathrm{D}$, respectivamente.

$$
\begin{aligned}
& \mathrm{PA}(\%)=\left(\frac{\mathrm{P}_{\mathrm{U}}-\mathrm{P}_{\mathrm{S}}}{\mathrm{P}_{\mathrm{U}}-\mathrm{P}_{\mathrm{I}}}\right) \cdot 100 \\
& \mathrm{AA}(\%)=\left(\frac{\mathrm{P}_{\mathrm{U}}-\mathrm{P}_{\mathrm{S}}}{\mathrm{P}_{\mathrm{S}}}\right) \cdot 100 \\
& \operatorname{RQ}(\%)=\left(\frac{\mathrm{L}_{1}-\mathrm{L}_{2}}{\mathrm{~L}_{1}}\right) \cdot 100 \\
& \operatorname{DA}\left(\mathrm{g} / \mathrm{cm}^{3}\right)=\frac{\mathrm{P}_{\mathrm{S}}}{\mathrm{P}_{\mathrm{U}}-\mathrm{P}_{\mathrm{I}}}
\end{aligned}
$$

nas quais Pu é o peso úmido, Ps o peso seco, Pi o peso imerso, L1 a altura do corpo de prova antes de ser sinterizado e L2 a altura do mesmo após sinterização. PA significa porosidade aparente, AA absorção de água, RQ é a retração linear após sinterização e DA é a densidade aparente. Para as pesagens foi utilizada uma balança analítica Gehaka BK400 acoplada com um kit de medição de densidade.

Para a resistência à compressão, utilizou-se a norma ASTM C1424 - 99 (Standard test method for monotonic compressive strength of advanced ceramics at ambient temperatures), com uma máquina universal de ensaios Emic
DL2000. O resultado foi obtido pela média aritmética de 5 corpos de prova. Os valores de resistência à compressão $\left(\mathrm{S}_{\mathrm{u}}\right)$ foram obtidos com a equação $\mathrm{E}$.

$$
\mathrm{S}_{\mathrm{u}}(\mathrm{MPa})=\frac{\mathrm{P}_{\text {máx }}}{\mathrm{A}}
$$

na qual $\mathrm{P}_{\text {máx }}$ é carga máxima (em N) e A a área de seção transversal inicial $\left(\mathrm{em} \mathrm{mm}^{2}\right)$ do corpo de prova.

Para o ensaio de micro-dureza, utilizou-se a norma C1327 - 99 (Standard test method for Vickers indentation of advanced ceramics). Para o ensaio de micro-dureza utilizouse um microdurômetro Shimadzu HMV Micro Hardness Tester. Foram realizadas 10 penetrações por amostra e o resultado obtido foi a média aritmética com desvio padrão das penetrações realizadas.

\section{RESULTADOS E DISCUSSÃO}

\section{Ensaios fisicos}

Foram avaliadas as seguintes características cerâmicas para este ensaio: porosidade aparente, absorção de água, densidade aparente e retração linear de queima. Os resultados obtidos para cada composição estão sumarizados na Tabela III.

Retração linear de sinterização - os valores médios variaram de $13,69 \%(\operatorname{amostra} \mathrm{C})$ a $20,71 \%(\mathrm{~A})$ a $1250{ }^{\circ} \mathrm{C}$; de $15,69 \%$ (B) a $26,43 \%$ (A) a $1300{ }^{\circ} \mathrm{C}$; e de $42,92 \%$ (B) a $45,69 \%$ (C) a $1350{ }^{\circ} \mathrm{C}$, o que indica um comportamento de retração linear semelhante para as 3 amostras a $1350^{\circ} \mathrm{C}$. A hidroxiapatita pura retraiu menos que as 3 amostras nas condições de temperatura de sinterização.

Absorção de água - os valores médios obtidos variaram de $21,77 \%$ (amostra C) a $42,96 \%$ (A) a $1250{ }^{\circ} \mathrm{C}$; de $23,52 \%$ (A) a $34,56 \%$ (B) a $1300{ }^{\circ} \mathrm{C}$; e de $8,29 \%$ (B) a $17,81 \%$ (A) a $1350{ }^{\circ} \mathrm{C}$. A hidroxiapatita pura absorveu mais água que as amostras nas três condições de temperatura.

Porosidade aparente - os valores médios obtidos variaram de $46,64 \%$ (amostra C) a $54,77 \%$ (B) a $1250{ }^{\circ} \mathrm{C}$; de $41,37 \%$ (A) a $50,85 \%$ (B) a $1300{ }^{\circ} \mathrm{C}$; e de $15,43 \%$ (B) a $35,68 \%$ (A) a $1350{ }^{\circ} \mathrm{C}$. Em termos de porosidade aparente, a hidroxiapatita pura não teve muita variação, o que indica que praticamente não há variação de porosidade aparente nas condições de temperatura de sinterização.

Densidade aparente - os valores médios obtidos variaram de $1,28 \mathrm{~g} / \mathrm{cm}^{3}$ (amostra B) a $1,51 \mathrm{~g} / \mathrm{cm}^{3}$ (A) a $1250^{\circ} \mathrm{C}$; de $1,48 \mathrm{~g} / \mathrm{cm}^{3}$ (B) a $1,77 \mathrm{~g} / \mathrm{cm}^{3}$ (A) a $1300{ }^{\circ} \mathrm{C}$; e de $2,04 \mathrm{~g} / \mathrm{cm}^{3}$ (A) a $2,49 \mathrm{~g} / \mathrm{cm}^{3}$ (B) a $1350{ }^{\circ} \mathrm{C}$. Levando-se em conta que a densidade teórica da hidroxiapatita $\mathrm{d}_{\mathrm{HA}}=3,156 \mathrm{~g} / \mathrm{cm}^{3}$ e que a da titânia $\mathrm{d}_{\text {titânia }}=4,35 \mathrm{~g} / \mathrm{cm}^{3}$, se as amostras fossem densas, deveriam ter densidades iguais a $3,753 \mathrm{~g} / \mathrm{cm}^{3}, 3,634 \mathrm{~g} / \mathrm{cm}^{3}$ e $3,514 \mathrm{~g} / \mathrm{cm}^{3}$ para as amostras A, B e C, respectivamente. Todas as amostras, independente da temperatura de sinterização, se mostraram menos densas, o que é uma característica de materiais porosos. A hidroxiapatita pura mostrou-se menos densa que os compósitos $\mathrm{A}$, B e C, nas temperaturas de sinterização. 
Tabela III - Resultados dos ensaios físicos para as amostras A, B e C, após sinterização a $1250{ }^{\circ} \mathrm{C}, 1300{ }^{\circ} \mathrm{C}$ e $1350{ }^{\circ} \mathrm{C}$. (Table III - Physical characteristics results for samples $\mathrm{A}, \mathrm{B}$ and $\mathrm{C}$ sintered at $1250^{\circ} \mathrm{C}, 1300^{\circ} \mathrm{C}$ and $1350{ }^{\circ} \mathrm{C}$.)

\begin{tabular}{|c|c|c|c|c|c|}
\hline \multirow{2}{*}{\multicolumn{2}{|c|}{ Amostra / temperatura $\left({ }^{\circ} \mathrm{C}\right)$}} & \multicolumn{4}{|c|}{ Propriedades físicas } \\
\hline & & RQ (\%) & $\mathrm{AA}(\%)$ & PA $(\%)$ & $\mathrm{DA}\left(\mathrm{g} / \mathrm{cm}^{3}\right)$ \\
\hline \multirow{3}{*}{ HÁ } & 1250 & $24,72 \pm 1,38$ & $51,57 \pm 18,05$ & $53,34 \pm 15,39$ & $1,07 \pm 0,14$ \\
\hline & 1300 & $29,91 \pm 6,96$ & $44,01 \pm 7,79$ & $51,42 \pm 4,79$ & $1,19 \pm 0,13$ \\
\hline & 1350 & $44,67 \pm 5,27$ & $39,68 \pm 3,17$ & $48,98 \pm 2,41$ & $1,24 \pm 0,05$ \\
\hline \multirow{3}{*}{ A } & 1250 & $20,71 \pm 5,03$ & $33,59 \pm 2,30$ & $50,69 \pm 2,02$ & $1,51 \pm 0,05$ \\
\hline & 1300 & $26,43 \pm 8,29$ & $23,52 \pm 2,52$ & $41,37 \pm 2,83$ & $1,77 \pm 0,07$ \\
\hline & 1350 & $43,61 \pm 5,11$ & $17,81 \pm 5,51$ & $35,68 \pm 8,08$ & $2,04 \pm 0,19$ \\
\hline \multirow{3}{*}{ B } & 1250 & $17,23 \pm 2,96$ & $42,96 \pm 5,75$ & $54,77 \pm 3,94$ & $1,28 \pm 0,08$ \\
\hline & 1300 & $15,69 \pm 3,59$ & $34,56 \pm 2,96$ & $50,85 \pm 2,54$ & $1,48 \pm 0,07$ \\
\hline & 1350 & $42,92 \pm 6,40$ & $8,29 \pm 3,04$ & $15,43 \pm 5,38$ & $2,49 \pm 0,18$ \\
\hline \multirow{3}{*}{$\mathrm{C}$} & 1250 & $13,69 \pm 4,66$ & $21,77 \pm 4,90$ & $46,64 \pm 4,76$ & $1,48 \pm 0,10$ \\
\hline & 1300 & $22,62 \pm 3,11$ & $32,94 \pm 5,58$ & $49,22 \pm 5,48$ & $1,51 \pm 0,10$ \\
\hline & 1350 & $45,69 \pm 5,23$ & $11,24 \pm 5,84$ & $22,52 \pm 11,63$ & $2,08 \pm 0,23$ \\
\hline
\end{tabular}

Tabela IV - Resultados do ensaio de compressão e dureza para as amostras A, B e C, sinterizadas a $1250{ }^{\circ} \mathrm{C}, 1300{ }^{\circ} \mathrm{C}$ e $1350{ }^{\circ} \mathrm{C}$.

(Table IV-Compression strength and hardness results for samples $A, B$ and $C$, sintered at $1250{ }^{\circ} \mathrm{C}, 1300^{\circ} \mathrm{C}$ and $1350{ }^{\circ} \mathrm{C}$.)

\begin{tabular}{cccccc}
\hline $\begin{array}{c}\text { Temperatura de } \\
\text { sinterização }\end{array}$ & $\begin{array}{c}\text { Propriedades } \\
\text { mecânicas }\end{array}$ & HA & A & B & C \\
\hline \multirow{2}{*}{$1250{ }^{\circ} \mathrm{C}$} & LRC (MPa) & $2,35 \pm 0,76$ & $5,54 \pm 1,15$ & $4,12 \pm 2,35$ & $3,19 \pm 0,79$ \\
& $\mathrm{HV}$ & $580 \pm 87,51$ & $709 \pm 91,48$ & $666 \pm 208,20$ & $620 \pm 131,23$ \\
\multirow{2}{*}{$1300^{\circ} \mathrm{C}$} & $\mathrm{LRC} \mathrm{(MPa)}$ & $1,97 \pm 1,05$ & $5,11 \pm 2,12$ & $4,65 \pm 0,82$ & $4,39 \pm 1,09$ \\
& $\mathrm{HV}$ & $654 \pm 82,68$ & $1084 \pm 312,30$ & $1064 \pm 351,36$ & $1017 \pm 369,05$ \\
$1350{ }^{\circ} \mathrm{C}$ & $\mathrm{LRC}(\mathrm{MPa})$ & $1,58 \pm 0,38$ & $14,64 \pm 1,51$ & $9,10 \pm 3,07$ & $9,05 \pm 2,04$ \\
& $\mathrm{HV}$ & $904 \pm 279,06$ & $1465 \pm 400,91$ & $1266 \pm 272,01$ & $1110 \pm 26,29$ \\
\hline
\end{tabular}

Foram avaliadas as seguintes características mecânicas para este ensaio: tensão de ruptura por compressão e microdureza Vickers. Os resultados encontram-se na Tabela IV. Os ensaios de resistência à compressão foram feitos a $34{ }^{\circ} \mathrm{C}$, com umidade relativa do ar $53 \%$, enquanto que os ensaios de dureza Vickers foram a $22,5^{\circ} \mathrm{C}$, com umidade relativa $60 \%$.

Limite de resistência à compressão-Os valores de limite de resistência à compressão da hidroxiapatita variaram de $1,58 \mathrm{MPa}\left(\right.$ a $\left.1350{ }^{\circ} \mathrm{C}\right)$ a $2,35 \mathrm{MPa}\left(1250^{\circ} \mathrm{C}\right)$, o que indica que à medida que a temperatura de sinterização aumenta, a resistência à compressão da hidroxiapatita diminui. Com a adição da titânia os valores do limite de resistência à compressão tiveram aumento nas 3 temperaturas de sinterização, sendo que o maior valor encontrado foi para a amostra A sinterizada a $1350{ }^{\circ} \mathrm{C}(14,64 \mathrm{MPa})$ e o menor valor foi para a amostra $\mathrm{C}$ sinterizada a $1250^{\circ} \mathrm{C}$. Em todas as condições do trabalho os valores obtidos para resistência à compressão foram maiores quando houve adição da titânia à hidroxiapatita que os valores dos corpos de prova confeccionados apenas com hidroxiapatita.
Dureza Vickers - Os valores de dureza Vickers para a hidroxiapatita variaram de $580 \mathrm{HV}\left(\right.$ a $1250{ }^{\circ} \mathrm{C}$ ) a 904 HV (a $1350{ }^{\circ} \mathrm{C}$ ), o que indica um aumento de dureza da hidroxiapatita a medida que a temperatura de sinterização aumenta. Com a adição de titânia à hidroxiapatita, os valores de dureza tiveram um aumento, sendo que o maior valor aconteceu para a amostra A sinterizada a $1350^{\circ} \mathrm{C}(1465 \mathrm{HV})$ e o menor valor de $620 \mathrm{HV}$ para a amostra $\mathrm{C}$ sinterizada a $1250{ }^{\circ} \mathrm{C}$. Em todas as condições do trabalho, houve aumento nos valores da dureza Vickers quando se acrescentou titânia à hidroxiapatita, nas proporções de $30 \%, 40 \%$ e $50 \%$ em massa.

\section{CONCLUSÕES}

A amostra A sinterizada a $1350{ }^{\circ} \mathrm{C}$ foi a que apresentou resultados com valores superiores de resistência mecânica à compressão e dureza Vickers em relação aos valores das demais amostras e aos da hidroxiapatita pura. A amostra A sinterizada a $1350{ }^{\circ} \mathrm{C}$ foi a que apresentou maior retração linear de queima. A hidroxiapatita pura praticamente teve 
valor de retração linear de queima semelhante ao da amostra A. A hidroxiapatita pura absorve mais água que as amostras A, B e C, independente da temperatura de sinterização. A amostra $\mathrm{A}$ foi a que absorveu menos água quando sinterizada a $1350{ }^{\circ} \mathrm{C}$. A amostra $\mathrm{B}$ foi a que teve menos porosidade aparente a $1350{ }^{\circ} \mathrm{C}$. A amostra A também apresentou valor baixo de porosidade aparente. A hidroxiapatita pura praticamente não teve variação de porosidade aparente; A amostra $\mathrm{A}$ sinterizada a $1350{ }^{\circ} \mathrm{C}$ foi a que obteve maior valor de densidade aparente, enquanto que a hidroxiapatita pura obteve menores valores. Para todas as amostras, os valores foram inferiores aos valores teóricos para amostras densas. Com estas conclusões, definiu-se que a melhor condição para os ensaios in vitro foi a da amostra A sinterizada a 1350 ${ }^{\circ} \mathrm{C}$, mesmo com redução do tamanho e quantidade de poros, além do aumento da densidade aparente.

\section{AGRADECIMENTOS}

Ao Conselho Nacional de Desenvolvimento Científico e Tecnológico $(\mathrm{CNPq})$ pela bolsa de fomento para doutorado.

\section{REFERÊNCIAS}

[1] R. L. Oréfice, M. M. Pereira, H. S. Mansur, Biomateriais: fundamentos e aplicações, Cultura Médica, Rio de Janeiro, RJ (2006) 59.

[2] B. D. Ratner, A. S. Hoffman, F. J. Schoen, J. E. Lemons, Biomaterials Science: An Introduction to Materials in Medicine, Academic Press, San Diego, EUA (1996) 73.

[3] D. M. Liu, Key Eng. Mater. 115 (1996) 209-232.

[4] L. R. Rodrigues, M. Motisuke, C. A. C. Zavaglia, $21^{\circ}$ Cong. Bras. Eng. Biomédica, Salvador, BA (2008) 1210 1213.

[5] D. A. Hirschfeld, T. K. Li, D. M. Liu, Key Eng. Mater. 115 (1996) 65-80.

[6] H. X. Peng, Z. Fan, J. R. G. Evans, J. J. C. Busfield, J. Eur. Ceram. Soc. 20 (2000) 807-813.

[7] M. H. Prado da Silva, A. F. Lemos, J. M. F. Ferreira, M. A. Lopes, J. D. Santos, Key Eng. Mater. 230 (2002) 483486.

[8] J. Saggio-Woyansky, C. E. Scott, Am. Ceram. Soc. Bull. 71 (1992) 1674-1682.

[9] H. T. Wang, X. Q. Liu, G. Y. Meng, Mater. Res. Bull. 32, 12 (1997) 1705-1712.

[10] X. Zhu, D. Jiang, S. Tan, Mater. Sci. Eng. A23 (2002) 232-238

[11] P. Souza Santos, Ciência e Tecnologia de Argilas, $2^{\mathrm{a}}$ Ed., Vol. 1, Edgard Blücher, S. Paulo, SP (1989) 194.

(Rec. 28/10/2011, Ac. 30/03/2012) 